\title{
GENERAL TRENDS IN THE EXECUTION OF DEFORMATION JOINTS AT THE ROAD BRIDGES WITH THE SIMPLE SUPPORTING ELEMENTS SUPERSTRUCTURE.
}

Mihai Netid

Master Degree

\section{Roads, Materials, and Mechanization in Construction}

\section{Road design engineer}

Abstract. The deformation joints are, along with the supports, the static structure of the bridge, desined to absorb the displacements, resulting from the movement of motor vehicles, strong winds, temperature range, and other unfavorable conditions. Joints have begun to be used with the construction of the first bridges with several openings. After this, with the enlargement of the openings and the begining of steel use for the construction of simple leaned elements, temperature deformations also increased, which led to the need of ensuring the necessary deformation joint.

Keywords: deformation joints, deck plate, joint cover device, exploitation.

\section{Deformation joints.}

To avoid negative effects of water penetration through the joints between the openings at simply supported deck plate, the rules allow allow them to be covered by achieving the continuity of the upper plate.

The joint cover device is an equipment which is mounted on the deck or at the ends of it (on deck and at marginal abutments) and ensures continuity of the path supporting at the same time the traffic effects, temperature disparity and long-term deformations of the concrete.

The joint cover device is a component part of the bridge construction. When they are not working properly, rapidly progressing the degradation of other elements, which immediately reduces the service life of the entire bridge construction and the comfort of the traffic participants. 
The deformation joints are elements loaded with static and dynamic loads. They are directly subjected to temperature actions, angular and linear displacements, but also after the contact with the vehicle tires, as well as combined actions. They must be resistant to both mechanical actions (dynamics, shocks, tiredness, polishing), physicochemical processes (reagents from snow removal, temperature changes).

The deformation joints to resist actions and loadings, it is necessary to meet the next requirements:

- Durability and reliability;

- Seal tightness (do not allow water to penetrate the lower elements);

- Absorption of temperature movements in different directions;

- Ensuring comfortable traffic conditions;

- Strength of the joint at the dynamic actions from traffic;

- Resistance of the construction at the contact with chemical elements.

On multi-opening superstructures, made of simple leaned reinforced concrete beams, it is ascertaining that on the joints between the openings cause degradation of concrete and reinforcements, and a very unpleasant exterior view, due to unsatisfactory solutions and above all, lack of maintenance (figure 1.1).

A solution to avoid these situations, allowed by the codes is that of covering the joint by continuation of the plate. In consequence, there appears a link between the two adjacent openings which can be double articulated or double recessed. It brings with it, some changes to the calculation mode [1].

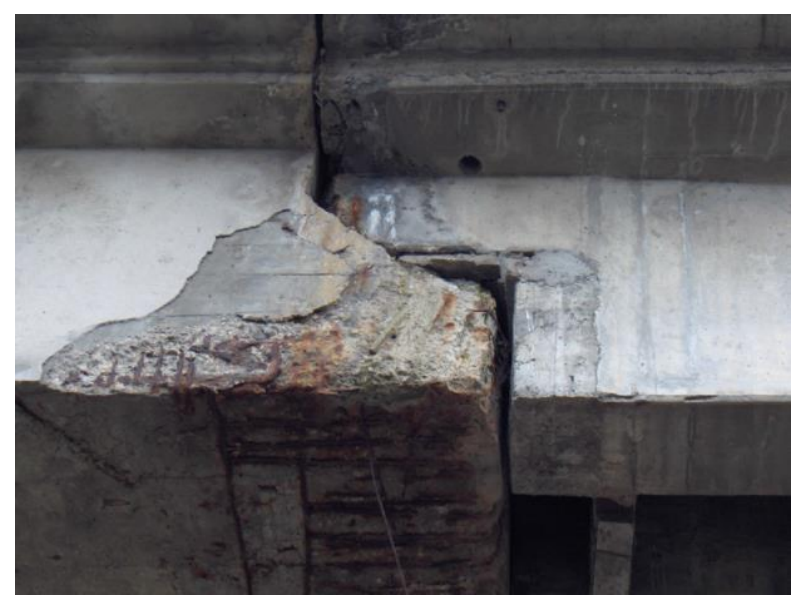


Fig. 1.1. Degraded expansion joint at the reinforced concrete bridge.

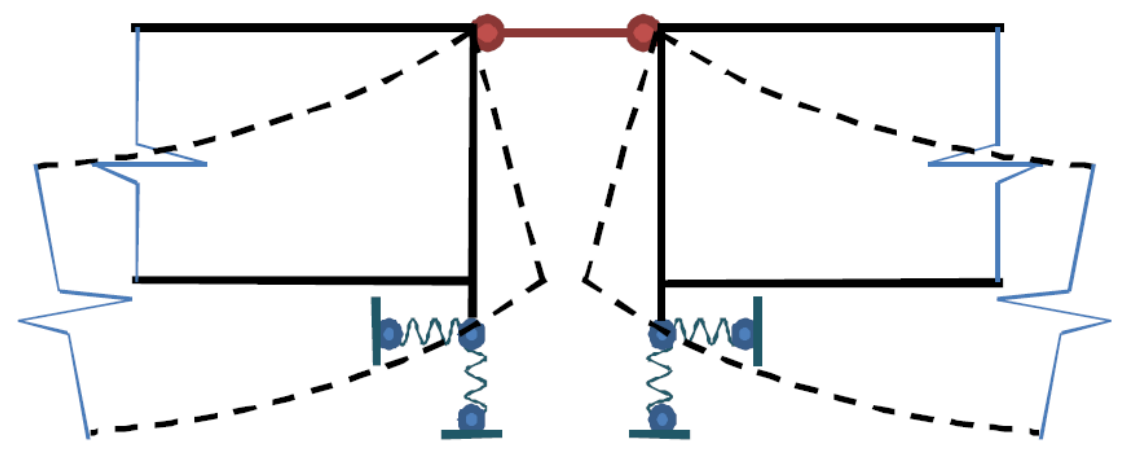

Fig. 1.2. Schematization of the plate continuity.

The expansion joint cover devices used in road bridges provide:

- Free movement of the bridge deck ends in the joints left for this purpose;

- Continuity of runway surface in joint area;

- Tightness at water leakage and infiltration.

Watertight devices are used to meet these requirements.

In general, the components of expansion joint cover device are [2]:

- Elastomeric elements which ensures the movement;

- Metallic support elements, fixed on structures;

- Special concrete in the catch area of the metal parts;

- Special sealing mortars;

- Rubber tapes for collecting and discharging the infiltration water.

Depending on the device type, the functionalities of some of the elements that come into their composition can be combined. The expansion joint cover devices are been applying to new bridges or to bridges in use, with specific gripping solutions for each case. If applied to bridges in use, the devices must allow the working process on a half of the way, and the movement of motor vehicles on the other side of the bridge without this technology compromising the technical features of the device. The expansion joint cover device includes all component elements, namely:

- Concrete in which metal elements are fixed; 
- Metal fasteners;

- Elastomeric element;

- Rubber sealing element;

- Special mortar for sealing the elastomeric element.

From traffic action, joint devices take over the effects of weight on tires, from braking effects on the bridge deck or on the joint device, as well as from rotation of the deck caused by the bending of the opening adjacent to the joint with the heavy actions. In determining the right joint type on the bridge superstructure, several aspects are important:

\section{1) The total deformation the device must take.}

The mobile support takes on the following loads:

- $\Delta \mathrm{l}_{1}-$ movement caused by temperature;

- $\Delta \mathrm{l}_{2}$ - from the deck rotation;

- $\Delta \mathrm{l}_{3}$ - from concrete contraction;

- $\Delta \mathrm{l}_{4}$ - slow flow of the concrete.

2) Mounting temperature of the equipment

Mounting temperature of the joint device to deck, should be $20-25^{\circ} \mathrm{C}$.

$$
\Delta l_{1}=l * \alpha * \Delta t,
$$

where: $l-$ the length at which it expands;

$\alpha-$ coefficient of expansion, $10^{-5}$

$$
\Delta l_{2}=\theta * h,
$$

where: $\theta$ - turning at expansion;

$h$ - beam height.

$$
\Delta l_{3,4}=f\left(E_{c}, t, \sigma_{c}, \text { concrete properties } \rightarrow \text { contraction, slow flow }\right)
$$

where: $t-$ the moment they will be calculated; 
$\sigma_{c}-$ the unitary effect in concrete.

\section{3) Type of support device from the edge of the deck which fits the joint}

Used joints at the road bridges:

- The gauge of the joint:

1) Low profile joints 10 (20) - $50 \mathrm{~mm}$;

2) Medium sized joints 50 - 100 (150) mm;

3) Large gauge joints $100(150)-500(800) \mathrm{mm}$.

Deformation joints are a component of great importance in the construction of the bridge. In the work refusal of the joint, the degradation of other elements quickly progresses, which quickly reduces the operation of the bridge construction and the comfort of the traffic participants.

Deformation joints are the bridge elements which absorb dynamics and statics loads. They are constantly subject to temperature actions, linear and angular displacements, as well as contact with vehicle tires, and the combined actions between them. They must resist both mechanical actions (dynamics, shocks, tiredness, polishing) and physicochemical actions (reagents from snow removal, temperature changes).

\section{Technical aspects and exploitation of the expansion-strain joints}

To resist the actions of the prejudicially factors, they must follow some strict requirements:

- Long service life and durability;

- Tightness ensuring (to prevent water penetration to infrastructure);

- Perception of temperature difference;

- Ensuring the traffic passes over the joints in comfort;

- Resistance of the joint to the dynamic actions of the road traffic;

- Resistance to chemical actions. 
The warranty period of the device is at least 10 years of normal operation of the bridge.

The elastomer element must be interchangeable. The guarantee term of the elastomer is at least 5 years.

During the warranty period, the company guaranteeing the device must provide its own repair or replacement and remedy the effects of structural damage due to faults of the device during this period.

The company delivering the device must ensure:

- Delivery of interchangeable elements, on request, for 30 years after the device has been put into operation;

- Providing specific small mechanical tools and equipment needed to operate the device and to change the elastomer element;

- Ensuring technical supervision when the device is instaled;

- Technical instructions for execution and exploitation.

The device must meet the following physico-mechanical characteristics in the temperature range $-35^{\circ} \mathrm{C} \div+80^{\circ} \mathrm{C}$ :

- Ensure free movement of the structure at the prescribed value;

- Metal fasteners must withstand corrosive agents;

- Be sealed;

- To be fixed to the structure of the bridge strength absorbing the vertical and horizontal actions. 


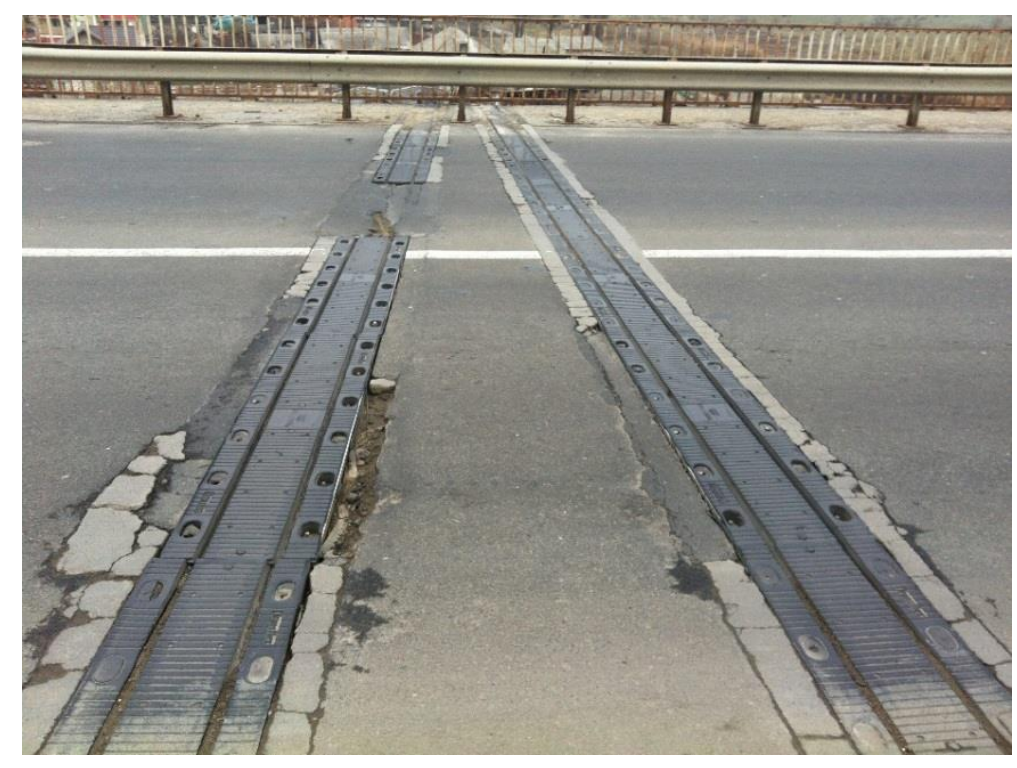

Fig. 2.1. Degradation of the joint device with the road pavement of the bridge construction

On the sides of the joint, on the road surface of the bridge, at the time of installation of the device must be executed and strictly adhered to the requirements prescribed by the producing company, because otherwise, as a result of the long-term exploitation and increased intensity of road traffic, degradation of the connections of this device with the road pavement of the bridge will be followed (fig. 2.1).

In the case of long-term exploitation (exceeded) of the expansion joints and inappropriate maintenance, especially in urban areas with increased traffic intensity (including heavy traffic), major degradations are observed both for the connection of the joint with the road pavement and the joint itself, where it is observed an uneven opening and increased here and there, as well as infiltration of mud and plant debris into the device.

In some cases, during the maintenance of the bridge construction, it is tried to cover the joint device with asphaltic mixture, but due to the large openings of the bridge construction it can be observed the dilation of the simple supported structure, because the elasticity of the asphalt concrete covering the joint does not ensure the opening due to the expansion of the concrete at temperature actions as well as shocks from heavy traffic [2]. 


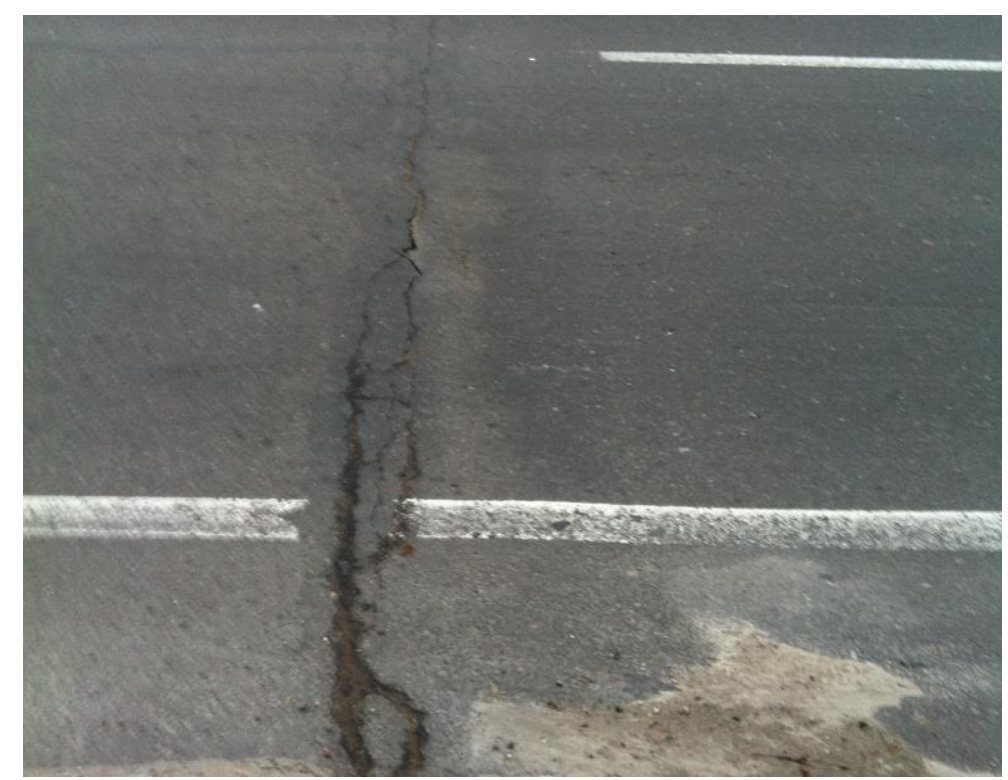

Fig. 2.2. Covering the joint device with asphalt mixture

An important factor in operating the devices is the work of any kind of deformation joint in extreme conditions - usually, construction of the bridge evacuation system and bridge approach holds both a longitudinal and a transverse gradient, and this means that, the water volume on the superstructure and the aproach roadway, is moving in the direction of the deformation joints. That is why deformation joints together with other effects support the actions of humidity which infiltrates through the cracks formed between the joint and the bridge's pavement. Once they get into the device, water worsens the concrete quality of the superstructure plate. This may continue to degrade the construction, deformation joint and superstructure plate. In addition to those listed, the construction of the deformation joint poses a risk of being damaged by the blades of the snow-cleaning technique, which leads to the reducing of the operating time under normal conditions.

\section{In conclusion}

After analyzing the current problems of jointing devices, a number of lacks have been observed both at the time of setting up the devices and for the operating period, which over time lead to higher repair costs, but also directly affect the bridge infrastructure, through the penetration of water and waste into it, which present an imminent danger to the safety of the road users and the construction bridge itself. 
Another problem that leads to degradation of joint cover devices is the wrong choice of the support device for a particular bridge construction, because these elements absorb the superstructure displacements.

A major problem for most of the bridges around the world, represents the lack of current and periodic maintenance of these devices, which with cause degradation to other elements from the infrastructure, namely: lack of cleaning the devices of mud and waste; the penetration of reagents and petroleum products into them; the possibility of touching the devices with the snowmachine blade, and others.

\section{References:}

1. Continuing the plates at bridge decks on simple lied reinforced concrete beams and calculating implications. Bulletin of the Polytechnic Institute from Iasi, Technical University "Gheorghe Asachi” from Iasi. Topa Nicolai, Ghindea Cristian -6 pages.

2. Road Bridges deformation joints: Features of construction and work. A.V.Efanov, I.G. Ovchinikov, V.I.Shesternikov, V.N.Makarov. Saratov 2005 -94 pages. 